\title{
Differential effects of pharmacologic stressors: More than meets the eye
}

\author{
Philipp A. Kaufmann, MD
}

\section{See related article, p. 324}

Pharmacologic stress for myocardial perfusion imaging or inducible wall motion abnormality assessment is an established and widely accepted alternative to physical exercise, and represents the method of choice in many patients with limited exercise capacity. The diagnostic performance of such testing may by subject to a substantial variability according to the combination of stress agents and respective imaging modality. ${ }^{1}$ Therefore, it is important to know the mechanism of action for each stressor to achieve the maximal clinical benefit for each patient. It generally is supported that pharmacologic stress by vasodilators such as adenosine and dipyridamole causes near-maximal hyperemic myocardial blood flow (MBF) response. This allows accurate detection of coronary artery disease (CAD) by inducing flow heterogeneities because flow increase in territories subtended by stenotic coronary arteries is blunted compared with remote segments. Only in a minority of patients and mainly in case of high-grade stenotic lesions may adenosine cause steal effects and induce real ischemia as evidenced by wall motion abnormalities. ${ }^{1}$ By contrast, dobutamine indirectly increases MBF by increasing myocardial contractility and consequently oxygen consumption, which needs to be matched by an increase in MBF, mainly mediated by the metabolic pathway. Again, this increase is blunted by stenotic lesions causing flow-response disparity between stenotic and remote segments indicative of CAD. In addition, the blunted increase to dobutamine stimulation may induce real ischemia as a result of the oxygen demand-supply mismatch. Because this mechanism appears to better mimic the physiologic situation of

From the Cardiovascular Center, Nuclear Cardiology, University Hospital Zurich, Zurich, Switzerland, ${ }^{\text {a }}$ and the Center for Integrative Human Physiology, University of Zurich, Zurich, Switzerland. ${ }^{b}$

Supported by the Swiss National Sciences Foundation (professorship grant no. PP00A-68835).

Reprint requests: Philipp A Kaufmann, MD, Swiss National Science Foundation, Professor of Nuclear Medicine and Cardiology, Director Nuclear Cardiology, University Hospital Zurich, NUK C 32, Ramistr. 100, CH-8091 Zurich, Switzerland; pak@usz.ch.

J Nucl Cardiol 2006;13:311-2.

$1071-3581 / \$ 32.00$

Copyright $(\odot 2006$ by the American Society of Nuclear Cardiology. doi:10.1016/j.nuclcard.2006.03.011 exercise-induced ischemia compared with vasodilators, one intuitively may expect diagnostic superiority of dobutamine and bicycle stress over vasodilator stress. Interestingly, however, in clinical trials adenosine proved at least equivalent to inotropic stressors. ${ }^{2-4}$

The study by Jagathesan et $\mathrm{al}^{5}$ adds to the mechanistic explanation of this observation. In 36 patients with single-vessel CAD and 18 healthy volunteers the response to either dobutamine or adenosine stress was assessed with positron emission tomography and ${ }^{15} \mathrm{O}$ labeled water. In healthy volunteers and in remote segments of CAD patients, adenosine achieved significantly greater hyperemia than dobutamine. The latter induced MBF responses comparing well with those reported for bicycle exercise in healthy volunteers ${ }^{6}$ and in $\mathrm{CAD}$ patients. ${ }^{7}$ In myocardial segments subtended by a significant coronary stenosis, there was progressive blunting of regional MBF with both agents. Surprisingly, however, dobutamine achieved greater hyperemia. This greater hyperemia with dobutamine may be owing to more pronounced collateral recruitment whereas the tendency of vasodilator agents to induce coronary steal may play a modest role. Consequently, flow disparity was greater with adenosine stress at all grades of coronary stenoses. This is in agreement with a recent metaanalysis on pharmacologic stress testing supporting the highest sensitivity and lowest specificity to be attained by use of vasodilator combined with myocardial perfusion imaging, while the highest specificity and lowest sensitivity to be attained by use of a vasodilator and an inducible wall motion abnormality. The lower diagnostic sensitivity attributed to dobutamine stress may be explained in part by less pronounced flow heterogeneities and in part by the higher sensitivity of abnormal perfusion over regional dysfunction as described by the ischemic cascade.

In addition to providing mechanistic insights, the results by Jagathesan et $\mathrm{al}^{5}$ are important for another reason. In contrast to the semiquantitative information on flow heterogeneity provided by single photon emission computed tomography, positron emission tomography provides absolute quantification of $\mathrm{MBF}$ at rest and during pharmacologic stress, allowing calculation of coronary flow reserve (CFR) as the ratio of stress over resting MBF. CFR is used as an integrated measure of flow through both the large epicardial coronary arteries 
and the microcirculation ${ }^{8}$ and has been proposed as an indirect parameter to evaluate the function of the coronary circulation. It is crucial to know the variance in CFR induced by different agents because Jagathesan et $\mathrm{al}^{5}$ showed that CFR values obtained with vasodilators are not interchangeable with those obtained with inotropic stimulation. Monitoring of treatment effects by CFR must be performed with the same stress agent to allow meaningful interpretation of CFR.

\section{Acknowledgment}

The author has indicated he has no financial conflicts of interest.

\section{References}

1. Paetsch I, Jahnke C, Wahl A, Gebker R, Neuss M, Fleck E, et al. Comparison of dobutamine stress magnetic resonance, adenosine stress magnetic resonance, and adenosine stress magnetic resonance perfusion. Circulation 2004;110:835-42.
2. Levine MG, Ahlberg AW, Mann A, White MP, McGill CC, Mendes de Leon $\mathrm{C}$, et al. Comparison of exercise, dipyridamole, adenosine, and dobutamine stress with the use of Tc-99m tetrofosmin tomographic imaging. J Nucl Cardiol 1999;6:389-96.

3. Wright GA, McDade M, Keeble W, Martin W, Hutton I. Quantitative gated SPECT myocardial perfusion imaging with 201Tl: an assessment of the limitations. Nucl Med Commun 2000;21:1147-51.

4. Shaw LJ, Hendel R, Borges-Neto S, Lauer MS, Alazraki N, Burnette J, et al. Prognostic value of normal exercise and adenosine (99m)Tc-tetrofosmin SPECT imaging: results from the multicenter registry of 4,728 patients. J Nucl Med 2003;44:134-9.

5. Jagathesan R, Barnes E, Rosen S, Foale R, Camici P. Comparison of myocardial blood flow and coronary flow reserve during dobutamine and adenosine: implications for pharmacologic stress testing in coronary artery disease. J Nucl Cardiol 2006;3:324-32.

6. Wyss CA, Koepfli P, Mikolajczyk K, Burger C, von Schulthess GK, Kaufmann PA. Bicycle exercise stress in PET for assessment of coronary flow reserve: repeatability and comparison with adenosine stress. J Nucl Med 2003;44:146-54.

7. Wyss CA, Koepfli P, Fretz G, Seebauer M, Schirlo C, Kaufmann PA. Influence of altitude exposure on coronary flow reserve. Circulation 2003;108:1202-7.

8. Kaufmann PA, Camici PG. Myocardial blood flow measurement by PET: technical aspects and clinical applications. J Nucl Med 2005;46:75-88 